\title{
POSITIVE NEAR-APPROXIMANTS AND SOME PROBLEMS OF HALMOS
}

\author{
BY RICHARD BOULDIN
}

Communicated by P. R. Halmos, July 27, 1973

In [5] P. R. Halmos called for an investigation of those nonnegative operators $P$ with the property that the distance from $P$ to a fixed operator $T$ is the same as the distance of $T$ to the set of nonnegative operators. Such a $P$ is a "positive approximant" of $T$. Halmos asked for the properties of such best approximating nonnegative operators when other norms besides the operator norm were used to compute distance. If $T=B+i C$, with $B=B^{*}, C=C^{*}$, then the formula $\|T\|\left\|^{2}=\right\| B^{2}+C^{2} \|$ defines a norm on the bounded operators with the pı operty that

$$
\|T\| \geqq\|T\| \geqq w(T) \geqq \frac{1}{2}\|T\|
$$

where $w(T)$ denotes the numerical radius of $T$. The distance from $T$ to the nonnegative operators is the same whether it is computed with the operator norm or with the new norm. A nonnegative operator which best approximates $T$ in the new norm is a "positive near-approximant." This name is motivated by the facts that every positive approximant is a positive near-approximant and a positive near-approximant frequently turns out to be a positive approximant, although that is not necessarily the case.

P. R. Halmos gave an ingenious argument which resulted in a device for computing the distance of $T$ to the nonnegative operators, denoted $\delta(T)$, and in a formula which defines a positive approximant of $T$ for any $T$. If $T=B+i C$, with $B=B^{*}, C=C^{*}$, then the Halmos positive approximant is $P_{0}=B+\left(\delta^{2}-C^{2}\right)^{1 / 2}$ where $\delta=\delta(T)$. In [1] we showed that $P_{0}$ is absolutely maximal for the positive approximants of $T$, that is $P \leqq P_{0}$ whenever $P \in \mathscr{P}(T)$ with $\mathscr{P}(T)$ denoting the positive approximants; we used this fact as a basis for constructing positive approximants. In [2] we showed that $P_{0}$ is absolutely maximal for the positive nearapproximants of $T$, denoted $\mathscr{P}^{\prime}(T)$, and from this we constructed positive near-approximants. We have now carried this approach to the point of

AMS (MOS) subject classifications (1970). Primary 47A55; Secondary 46B99.

Key words and phrases. Nonnegative operator, best approximation, normal operator, positive approximant, convex, extreme point. 
determining much of the convex structure of the two convex sets $\mathscr{P}(T)$ and $\mathscr{P}^{\prime}(T)$, and it is these results which are described in this announcement. We denote the underlying complex Hilbert space by $H$ and the range of the operator $A$ is written $A H$.

THEOREM 1. Let $\mathscr{P}^{\prime}(T)$ denote the convex set of positive near-approximants of the normal operator $T$ and let $H_{0}$ denote the subspace

$$
\left(P_{0} H\right)^{-} \cap\left(\left(\delta^{2}-C^{2}\right) H\right)^{-} \text {. }
$$

If the dimension of $\mathrm{H}_{0}$ is denoted by $\mathrm{p}$ then we have

$$
\operatorname{dim} \mathscr{P}^{\prime}(T)=p^{2} .
$$

Here all infinite cardinal numbers are identified.

COROLlaRY 1. The normal $T$ has a unique positive near-approximant if and only if the closed span of the kernels of $P_{0}$ and of $\left(\delta^{2}-C^{2}\right)$ is all of $H$.

Corollary 2. Let $\mathscr{P}(T)$ denote the convex set of positive approximants of $T$ and let $p$ denote the dimension of $H_{0}$. Assume that either (i) or (ii) below holds:

(i) $T$ is normal,

(ii) $C$ and $C^{2}$ have the same commutant where $T=B+i C$ with $B=B^{*}$, $C=C^{*}$.

Then we have $\operatorname{dim} \mathscr{P}^{\prime}(T)=\operatorname{dim} \mathscr{P}(T)=p^{2}$.

In the event that $T$ is normal the distance $\delta(T)$ is given by a simple formula, namely $\delta(T)=\left\|B_{-}+i C\right\|$, where $T=B+i C, B=B^{*}, C=C^{*}$ and $2 B_{-}=\left(B^{2}\right)^{1 / 2}-B$. Halmos first proved this with a matrix argument. In [1] we showed that $T$ has a unique positive approximant if and only if every point of the spectrum of $T$ has a distance of $\delta$ to the nonnegative reals. We can now give a very quick proof of both of these facts. Let $\mathscr{A}$ denote the $C^{*}$ algebra generated by $T$ and let $C(\sigma(T))$ denote space of continuous functions on the spectrum of $T$ with the usual norm. The Gelfond transform, denoted $\Gamma$, gives an isometric isomorphism of $\mathscr{A}$ onto $C(\sigma(T))$ and $(\Gamma T)(z)=z$. (See paragraphs 4.30 and 4.31, pp. 93-94, of [4].) The positive part of the real part of $T$, denoted $B_{+}$, and the Halmos positive approximant belong to $\mathscr{A}$ and clearly the corresponding continuous functions are $\varphi(z)=x$ and $\psi(z)=x+\left(\delta^{2}-y^{2}\right)^{1 / 2}$, respectively, where $z=x+i y$ with $x$ and $y$ real. By considering the right and left half planes separately one easily sees that

$$
\|z-p(z)\|^{2}=\operatorname{supremum}\left\{\left(x_{-}\right)^{2}+y^{2}: z \in \sigma(T)\right\}
$$

where $p(z)$ is any nonnegative valued function continuous on $\sigma(T)$. By the spectral mapping theorem and the observation that $B_{-}+i C$ is a 
normal operator we obtain $\|z-p(z)\| \geqq\left\|B_{-}+i C\right\|$. From this inequality it is immediate that $\delta(T)=\left\|B_{-}+i C\right\|$ and $B_{+}$is a positive approximant for $T$.

One easily verifies that the distance from $z$ to the nonnegative reals is $d$ if and only if we have

$$
-x_{-}+\left(d^{2}-y^{2}\right)^{1 / 2}=0 .
$$

Since $B_{+}$is a positive approximant of $T$ and $\sigma(T)$ is compact there is a point $z_{0}$ in $\sigma(T)$ such that

$$
\delta=\left|z_{0}-\varphi\left(z_{0}\right)\right|=\left|-\left(x_{0}\right)_{-}+i y_{0}\right| .
$$

Consequently, if every point of $\sigma(T)$ has a distance of $d$ to the nonnegative reals then $d=\delta$. One now sees that $(*)$ above is equivalent to saying that $\varphi(z)$ and $\psi(z)$ coincide, or $B_{+}$and $P_{0}$ coincide. We have given an elementary argument in [2] to show that this implies

$$
\mathscr{P}^{\prime}(T)=\mathscr{P}(T)=\left\{P_{0}\right\} .
$$

On the other hand, if $T$ has a unique positive approximant then $P_{0}$ equals $B_{+}$and $\varphi(z)$ coincides with $\psi(z)$. This is equivalent to the assertion that every point of $\sigma(T)$ has distance $\delta$ to the nonnegative reals.

The very commutative approach of the above two paragraphs produces very little beyond the above proofs. The one further fact that can be deduced by this approach is that $\mathscr{P}(T)$, for $T$ a normal operator, is a finite dimensional convex set only if $\sigma(T)$ has only finitely many points with distance to the nonnegative reals not equal to $\delta$. In contrast the very elaborate constructive approach which produced Theorem 1 and its corollaries yields further information. An important preliminary fact is that $\mathscr{P}(T)$ and $\mathscr{P}^{\prime}(T)$ are both compact in the weak operator topology and consequently each is the closed convex hull of its extreme points by the Krein-Milman Theorem.

Theorem 2. Assume that $T$ is normal and that $H_{0}$ (as defined in Theorem 1) is finite dimensional. Define the operator $A_{0}$ by the formula

$$
2 A_{0}=P_{0}+R-\left|P_{0}-R\right|
$$

where $R=2\left(\delta^{2}-C^{2}\right)^{1 / 2}$. Then $A_{0}, P_{0}$ and $C$ commute and each is reduced by $H_{0}$. Let $\left\{e_{1}, \cdots, e_{p}\right\}$ be an orthonormal basis for $H_{0}$ which simultaneously diagonalizes the restrictions of $A_{0}, P_{0}$ and $C$; let $Q$ be the orthogonal projection onto $e_{j}$ for some $j=1, \cdots, p$. Then $P_{0}-A_{0} Q$ is an extreme point of each of the sets $\mathscr{P}(T)$ and $\mathscr{P}^{\prime}(T)$.

Corollary. Assume that $T$ is normal and that $H_{0}$ is a one dimensional subspace. Let $f_{0}$ be a unit vector in $H_{0}$ and let $\lambda_{0}$ and $A_{1}$ be defined by the 
equations

$$
\lambda_{0}=\min \left\{\left\langle P_{0} f_{0}, f_{0}\right\rangle,\left\langle 2\left(\delta^{2}-C^{2}\right)^{1 / 2} f_{0}, f_{0}\right\rangle\right\}, \quad A_{1}=\left\langle\cdot, f_{0}\right\rangle \lambda_{0} f_{0} .
$$

Then $\mathscr{P}(T)$ and $\mathscr{P}^{\prime}(T)$ coincide with the convex hull of $P_{0}$ and $P_{0}-A_{1}$; consequently we have

$$
\mathscr{P}^{\prime}(T)=\mathscr{P}(T)=\left\{P_{0}-\lambda A_{1}: \lambda \in[0,1]\right\} .
$$

The detailed proofs of these results will appear elsewhere.

\section{REFERENCES}

1. R. H. Bouldin, Positive approximants, Trans. Amer. Math. Soc. 177 (1973), 391-403.

2. - Operators with a unique positive near-approximant, Indiana Univ. Math. J. (to appear).

3. - The convex structure of the set of positive approximants for a given operator (to appear).

4. R. G. Douglas, Banach algebra techniques in operator theory, Academic Press, New York, 1972.

5. P. R. Halmos, Positive approximants of operators, Indiana Univ. Math. J. 21 (1971/72), 951-960. MR 45 \#919.

Department of Mathematics, University of Georgia, Athens, Georgia 30601 